
https://doi.org/10.30534/ijeter/2019/037102019

\title{
An Emperical Study on Support Vector Machines for Intrusion Detection
}

\author{
N. Chandra Sekhar Reddy ${ }^{1}$, Purna Chandra Rao Vemuri ${ }^{2}$, A. Govardhan ${ }^{3}$ \\ 1,2 Department of Computer Science and Engineering, MLR Institute of Technology, Hyderabad, 500043,India. \\ naguchinnI@gmail.com, pcrao.vemuri@gmail.com \\ 3Professor \& Rector, Department of Computer Science and Engineering, JNTUH, Hyderabad, India. \\ govardhan_cse@jntuh.ac.in
}

\begin{abstract}
In today's world, tera-bytes of data are stored and exchanged starting from one source to another destination and vice-versa. The information when exchanged or stored over the network, there is possibility to attacks and tampered. Different procedures or applications are accessible to secure information from existed vulnerability. In this way we can analyze the information and to decide several algorithms have been proposed which might be emerged to mitigate vulnerabilities. This paper surveys machine learning algorithm SVM that has been implemented for classification and regression. Related to networking, Lot of researches are done that proves SVM performs efficient performance in simplification of network issues when compare to other network classifiers. Our research paper shows a speculative part of SVM with its concepts and applications.
\end{abstract}

Key words : KDD Cup'99, Machine Learning, Network Traffic Classification, NSL KDD, Support Vector Machine.

\section{INTRODUCTION}

Anomaly Detection System are implemented to protect from the exploit attack when information is exchanged over network. An Intrusion Detection System is an approach for identifying, analyzing and categorizing the network traffic data. An Intrusion Detection System (IDS) is an application or gadget used to assemble and analyze data that goes over a system or host. An IDS is intended to analyze, distinguish, and provide details regarding any infringement or abuse of a system or host. An IDS is utilized to screen and secure systems and networks by recognizing malicious action and announcing it to a gathering or contact, for example, a network/system administrator. When exercises of this sort are distinguished, an administrator is cautioned [1]. An IDS is intended to recognize malicious or non-standard conduct and assembles data from inside a system to distinguish infringement of security arrangement and furthermore report infringement and deviations to a director or framework proprietor [2]. In most recent decade, feature extraction has turned into a mainstream region of research in technology and a standout amongst the best utilizations of picture examination, categorization and comprehension.IDS works based on machine-learning algorithms, for instance SVM. SVM-Support Vector Machines are developed in 1990's and mostly used for recognition of text, speech and image. Basically, machine learning algorithms like SVM are applied to huge sample categorization issues such as text classification, image recognition, appearance exposure, speech recognition, pattern-matching, and defective card exposure, etc. Sample categorization intents to categorize information data in light of priori data and measurable data which taken from unprocessed information data.

\subsection{NETWORK TRAFFIC ATTACKS}

Now let's discuss about the widely known attacks, redundant records and rough attack distribution which causes trouble for identification in ID systems. These attacks can be reduced by identifying attack features and categorization of features so that it should not suffer from any of the troubled attacks. SVM, machine learning algorithm which involves in feature identification and feature categorization in network traffic analyzing data in Single connection vectors in and noticed either ordinary or an attack, with specifically on exact attack category. The represented attacks fall in one of the accompanying four categories [3].

\subsubsection{Probing Attack}

To find information about the target, sometimes, passive reconnaissance may not work in such cases the attacker has to send specifically crafted packets to get information. This is also referred as active scanning methodology from the perspective of hackers, the information what we will get here will have more possibilities of getting caught. For instance: Port scanning.

\subsubsection{DoS-Denial of Service Attack:}

Denial-of-Service Attacks are used by the flood system users or network unusable or an organization's system. These attacks can be considered against the availability of the user data that significantly slows the system by overloading resources or preventing authorized users from accessing the system. For instance: SYN flood, Ping of Death. 


\subsubsection{U2R-User to Root Attack:}

$\mathrm{U} 2 \mathrm{R}$ is an attack type where attacker already has control over the device, but s/he could not accomplish everything s/he wishes to. So, the attacker will exploit internal vulnerabilities to get more control or often called as root access of the device. This method will also be referred as privilege escalation. For instance: Memory Overflow Attack or Stack Overflow Attack.

\subsubsection{R2L-Remote to Local Attack:}

When user is not a valid user of the specific computer or the user is not a part of the internal network, he might try some exploitation to get access to the device from another network or another computer by sending some malicious packets to that computer. For instance: password guessing.

\section{SVM CLASSIFIER HISTORY}

SVM is first constructed in earlier 1990's, which is part of machine learning algorithms for information analysis based on statistical and quadratic programming.SVM became popular after it is constructed by famous authors Boser, Guyon and Vapnik in COLT-1992 and also by Cortes and Vapnik in 1995[4]. Theoretically SVM is a Well-driven algorithm which emerged from statistical learning theory by authors Vapnik and Chervonenkis since 1990[5]. Many applications in different fields like bioinformatics, text, image and pattern recognition are successful.

In remote sensing, SVM was basically implemented for the hyperspectral picture grouping and object identification (Gualtieri, 1999[6]; Melgani and Bruzzone, 2004[7]); in spite of the fact that researchers have recently improving its implementation towards multispectral remote detecting information (Mathur and Foody [8] and Huang [9]; Pal and Mather [10]). Melgani and Bruzzone[7] and Huang [9] stated a clearer intro of SVM to the remote sensing community. Mountrakis[1] encapsulated experimental outcomes from more than 100 articles with the help of SVM image classification algorithm. The main feature of SVM was great generalization efficiency with reduced training samples and computational prerequisites. Be that as it may, SVM gave better execution looked at then most other picture characterization calculations for both certifiable remote detecting information and recreated tests. With hyperspectral remote sensing information, Melgani, and Bruzzone (2004) [7] conducted a full comparison of SVM, usual K-nearest neighbor, and radial basis neural network. Their outcomes stated that SVM substantially defeated both the other classifiers. They came up with a point that SVM was somewhat reduced sensitivity to the Hughes phenomenon, as a result picking features flow may not be required for high dimensional datasets. In addition to that, they matched a collection of SVM multi-class grouping plans including single-to-single, single-to-multi and Ordered tree-based classification approaches or scheme. The outcomes from these methods seems to be very similar. The major performance from SVM was also reported by (Camps-valls[12], Bruzzone and Camps-valls, 2005[13], Gualtieri, 1999[6]), precisely relating to the classification of hyperspectral remote detecting data.

Usually, SVM preparation time scales quadratic-ally (or more awful) in the quantity of illustrations, so looks into endeavor all the ideal opportunity for more productive preparing algorithms. Generally, in arched quadratic optimization issues are solvable in polynomial time; it is hard to complete in practice. whereas SVM training datasets related to network are used in lot of issues to reduce the time consumption for solving the issue. Raised advancement issues have been widely considered, a huge number of algorithms have been proposed. Prior, SVMs were prepared with instant programming bundle, for example, MINOS, LOQO and MATLAB [14]. Machine-learning algorithm, SVM is a supervised type which categorizes each identifiable as belonging to one of the many categories from the given list of instruction sample examples. An SVM constructs a typical method that estimates the category of new sample cases. For this kind of classifications SVM has much capability to simplify the issue, that meant the aim of statistical learning method [15].

The statistical learning theory suggests intended for precising issues of learning ability, estimation planning and building choices from a collection of records. It allows selecting of hyper plane space according to its closest which represents the basic function in finalized space. Basically, in statistical algorithm's states the difficulty of machine learning is figured as follows. Here, we are assumed a collection of training records $\left\{\left(\mathrm{x}_{1}, \mathrm{y}_{1}\right) \ldots\left(\mathrm{x}_{\mathrm{n}}, \mathrm{y}_{\mathrm{n}}\right)\right\}$ in $\mathrm{R}^{\mathrm{n}} \mathrm{x} \mathrm{R}$ experimented, according to indefinite probability distribution(PD) $\mathrm{P}(\mathrm{x}, \mathrm{y})$, and a loss function $\mathrm{L}(\mathrm{y}, \mathrm{f}(\mathrm{x}))$ calculates the error, for a definite value of $\mathrm{x}, \mathrm{f}(\mathrm{x})$ is "predicted" rather than the actual value of $y$.

finding a function ' $\mathrm{f}$ ' that reduces the expected error Finding a function ' $\mathrm{f}$ ' that reduces the occurrence of error on newly added data. [16]

$\int P(x, y) L(y, f(x)) d x d y \quad$ eq:1.

In $20^{\text {th }}$ century, to learn the representation of unsophisticated methods, machine learning algorithms are mostly used. From there it became aim of learning to provide a proposition that performs accurate categorizations of training data records. The capability of a proposition to provide accurate categorize data records but not in training records.

\subsection{SVM MODEL FOR INTRUSION ESTIMATION}

In our paper, we construct a model of SVM for categorization/arrangement. SVM can capable of solving the issue of linear inseparability and pattern recognition. SVM will identifies the intruders or intrusions when network traffic 
issues occur by attacks. SVM will influence utilization of a HD space which is utilized to discover a hyper plane, to process the arrangement/classification for minimizing the occurrence of error. The SVM algorithm also used to train Intrusion Detection System and identifying multiple support vectors by using a part of information sets called training data. Thus, SVM forms several support vectors that represents a category or arrangement

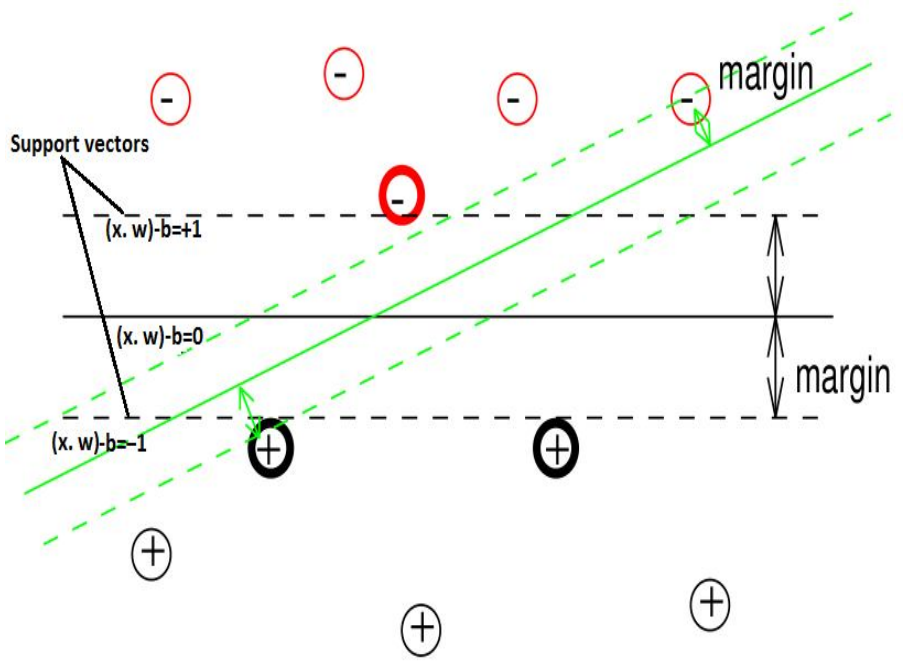

Figure 1: A Model of SVM ${ }^{17}$

By this model, SVM can categorize a specified document either known or unknown. A sample input data factors and output information factors are exposed as follows

$\left(\mathrm{p}_{1}, \mathrm{q}_{1}\right)\left(\mathrm{p}_{2}, \mathrm{q}_{2}\right) \ldots \ldots \ldots \ldots \ldots\left(\mathrm{p}_{\mathrm{n}}, \mathrm{q}_{\mathrm{n}}\right) \mathrm{p} \in \boldsymbol{R}_{\mathrm{T}} \mathrm{q} \in\{+1,-1\}$

Whereas,

$\left(\mathrm{p}_{1}, \mathrm{q}_{1}\right)\left(\mathrm{p}_{2}, \mathrm{q}_{2}\right) \ldots \ldots \ldots \ldots \ldots\left(\mathrm{p}_{\mathrm{n}}, \mathrm{q}_{\mathrm{n}}\right)$ are training data,

' $\mathrm{n}$ ' is no. of samples,

' $\mathrm{T}$ ' is input vectors.

On linear problems, a hyper-plane can be isolated into two distinct sorts as shown in Figure 1.

The Hyper Plane condition is

$$
\text { (x. } w)-b=0 \quad \text { eq: } 2
$$

And two categories are formulated as follows:

$$
\begin{array}{ll}
\text { (x. w)-b }=+1 & \text { eq:3 } \\
\text { (x. w)-b }=-1 & \text { eq:4 }
\end{array}
$$

\subsection{SVM's Basic Concept :}

Though, there are huge problems which are not simple to identify hyper planes to categorize the training records. Support Vector Machine normally manages design characterization that implies a calculation is utilized for the most part to classify the distinctive sorts of examples. Presently, there is distinctive kind of examples i.e. Direct and non-direct. Direct examples are designs that

are effortlessly recognizable or can be effectively isolated in low measurement while non-direct examples are designs that are not effectively discernable or can't be effectively isolated and consequently these sorts of examples should be additionally controlled with the goal that they can be effectively isolated.

In essence, SVM major principle is development of an ideal hyper-plane, can be used for categorized and for linear inseparable examples. An Ideal hyper plane which maximizes the margin of the hyper plane when it is selected for categorizing the patterns that is the space from the hyper-plane to adjacent patterns of every example. SVM aims to increase the margin for correctly categorizing the given data records.

The Hyper Plane Condition appeared beneath is [17]:

$$
\mathrm{pX}+\mathrm{qY}=\mathrm{R} \quad \text { eq:5 }
$$

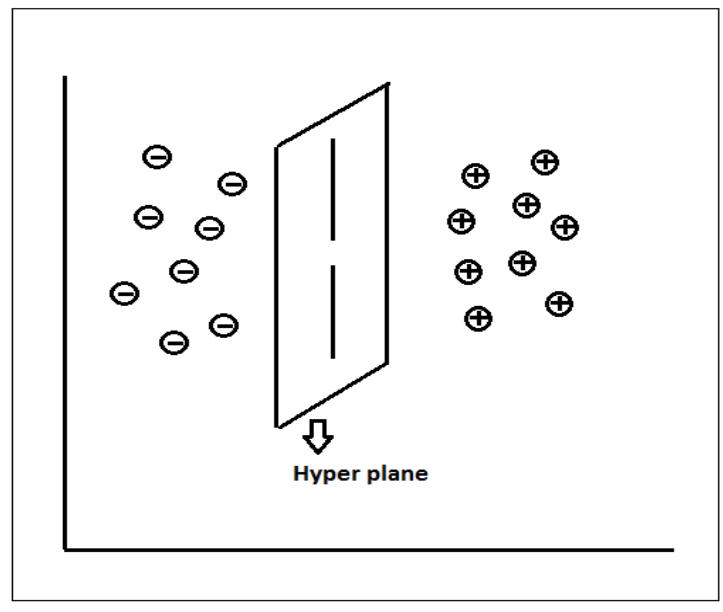

Figure 2: Hyper-Plane ${ }^{17}$

The above figure 2 is the fundamental thought of the hyper plane portraying what it would seem that like when two distinct examples are isolated utilizing a hyper-plane in 3-Dimension. Hyper-plane includes three categorized lines that isolates two distinctive in 3-D space, where one is known as marginal line and other two lines are support vectors.

SVM allows users to utilize numerous kernel functions to resolve different issues. we can resolve issue of linear-inseparability by selecting suitable kernel functions. SVM also capable of solving the issue of linear inseparability. Categorization function might be affected by internal productive processes. We can solve specified linear-inseparable issues by an appropriate kernel function $\mathrm{K}$ $\left(\mathrm{P}_{\mathrm{i}}, \mathrm{Q}_{\mathrm{j}}\right)$ without maximizing the complexity of operations. SVM classification has a major characteristic is selecting multiple kernel functions which are frequently used includes Radial Basic Function(RBF), LINEAR, POLYNOMIAL, AND SIGMOID. 
Several Kernel functions makes multiple plans for making non-direct partition shells. From equation5, Parameter ' $R$ ' is an imperative parameter in SVM algorithm, is termed as an unpredictable parameter and it is cumulative of partition of all other side focuses which are on wrong-side of hyper-plane. In general, during categorization process, an unpredictable parameter is measure of error which is disregarded. In any case, the estimation of arrangement process can't be too little. If the estimation of unpredictable constraint is very small at that point the execution of categorization is high.

The major theory behind SVM is that given collection of self-determining and indistinguishable circular training example: $\left(\mathrm{p}_{1}, \quad \mathrm{q}_{1}\right)\left(\mathrm{p}_{2}, \quad \mathrm{q}_{2}\right) \quad \ldots \ldots \ldots \ldots \ldots\left(\mathrm{p}_{\mathrm{n}}, \quad \mathrm{q}_{\mathrm{n}}\right)$ $\mathrm{p} \in R \mathrm{Rq} \in\{+1,-1\}_{\text {that }}$ implies $\left\{\left(\mathrm{p}_{\mathrm{i}}, \mathrm{q}_{\mathrm{i}}\right)^{\mathrm{N}}\right\}$, where $\mathrm{i}=1,2$, $\ldots \mathrm{n}, \mathrm{p} \in R_{\mathrm{rq}} \in\{+1,-1\}$, which indicates the input information and yield of the categorization.

The main objective of SVM remains in identification a hyper-plane (x. w)-b $=0$, that isolates two unique examples precisely. Thus, the issue of demonstrating ideal categorization which now converts into solving of quadratic programming issues. Here we have to check a partition of hyper-plane to create the two-sided blank zone $(2 /|\mathrm{w}|)$ extreme, that means we have to amplify the margin weight.

It is formulated as:

$\operatorname{Min} K(w)=1 / 2|w|^{2}=1 / 2(w, w), \quad$ eq: 6

Such that: $\quad \mathrm{q}_{i}((\mathrm{x} . \mathrm{w})-\mathrm{b})>=1 . \quad$ eq:7

\section{SVM APPLICATIONS}

- A demonstrative application case of classification using SVMs by Drucker. The paper "SVMs for spam characterization" by Drucker shows a group of constructing decisions which made in improvements of classifiers and also compares SVMs with another three algorithms which includes Boosting Decision Tree, Ripper, and Rocchio. At last this paper analysis and provides study and observation outcomes is exhibited [18].

- The paper "SVM's for Histogram-Based Picture Classification," by Chapelle shows a strategy for picture illustration using a histogram of shading parts. SVMs with kernel functions includes Radial Basic Function(RBF), LINEAR, POLYNOMIAL, AND SIGMOID are contrasted with a k-nearest neighbor strategy [18].

- These are an arrangement of related regulated learning techniques utilized for order and relapse. SVM is generally connected to data records of example acknowledgment. SVM is likewise utilized for a disruption discovery framework.

- SVM describes face to face character confirmation issues by using support vector machines. Face and discourse are two modalities for character confirmation. Observation results are exhibited for various distinctive paired classification strategies [18].

- Basically, in the single class SVM depends on one arrangement of illustrations having a place with a specific class and no negative cases as opposed to utilizing positive and negative case.

- When contrasted with neural systems in KDD 43 informational collection, it was discovered that SVM out performed Neural Networks (NN) as far as false caution rate and exactness in most sort of assaults.

- SVM also applied in cloud computing with two major classes as follows, One Class Support Vector Machines are utilized for identifying anomaly score, and Two Class Support Vector Machines are utilized for controlled when certain new information records are incorporated into the current dataset. [19]

- SVM order and k-medoids bunching for gathering comparable information cases by k-medoids method and coming about groups renamed into utilizing SVM classifiers. [19]

- Machine learning algorithm, Support Vector Machine is an administered type which performs on preparing premise, so it has for the most part been improvised in network regions. For E.g.: categorizing the distinctive system application protocols like file transfer protocol (FTP), Hyper-text transfer protocol (HTTP), Peer to Peer (P2P), and Substitute works of SVM examples are Text grouping, Verbal acknowledgment, Picture categorization, and numerous different applications that requires design acknowledgment system [20].

- The SVM can likewise be actualized in BOTNET recognition for confinement of vindictive activity, for development in arrange movement security. Additionally, a few works can be executed utilizing SVM by separating system movement to improve Quality of Service(QoS)[21].

\section{CONCLUSION}

From our study, SVM is capable for classifying the datasets such as KDD and NSL which are associated to network traffic. We can also filter out authorized datasets from anomaly datasets by detecting anomaly network traffic by using feature-based selection algorithms such as SVM, LS-SVM etc. These algorithms are also used for detecting network traffic like ping attacks, probe attacks, denial of service attacks, flood attacks, and distributed denial of Service attack. SVM is most widely used classifier for classifying network-based attacks which is practically implemented on various datasets. The results obtained from various research analysis proves that SVM can be combined with many other feature extraction techniques for effective intrusion detection.

\section{REFERENCES}

1. H. Debar et. al., Towards a taxonomy of intrusion detection systems, Computer Network, pp. 805-822, April 1999.

https://doi.org/10.1016/S1389-1286(98)00017-6 
N. Chandra Sekhar Reddy et al., International Journal of Emerging Trends in Engineering Research, 7(10), October 2019,383 - 387

2. Sundaram, A., An Introduction to Intrusion Detection, Crossroads: The ACM student magazine, 2(4), 1996. https://doi.org/10.1145/332159.332161

3. Rupali Datti, Shilpa Lakhina. Performance Comparison of Features Reduction Techniques for Intrusion Detection System, ISSN: 0976-8491 (Online) | ISSN: 2229-4333, IJCST Vol. 3, Issue 1, Jan. - March 2012.

4. Cortes \& Vapnik. The Nature of Statistical Learning Theory. New York: Springer-Verlag, 1995. https://doi.org/10.1007/978-1-4757-2440-0

5. V. Vapnik and A. Chervonenkis. On the uniform convergence of relative frequencies of events to their probabilities, Doklady AcademiiNauk USSR, vol. 181, no. $4,1968$.

6. Gualtieri, J.A., Cromp, R.F., 1999. Support vector machines for hyperspectral remote sensing classification. Proc. SPIE 27th AIPR Workshop:Advances in Computer Assisted Recognition,Washingtong DC, 14-16 October, pp. 221-232.

https://doi.org/10.1117/12.339824

7. Melgani, F., Bruzzone, L., 2004. Classification of hyperspectral remote sensing images with support vector machines. IEEE Transactions on Geoscience and Remote Sensing 42 (8), 1778-1790.

8. Foody, G.M., Mathur, A., 2004. A relative evaluation of multiclass image classification by support vector machines. IEEE Transactions on Geoscience and Remote Sensing 42 (6), 1335-1343. https://doi.org/10.1109/TGRS.2004.827257

9. Huang, C., Davis, L.S., Townshend, J.R.G., 2002. An assessment of support vector machines forland cover classification. International Journal of Remote Sensing 23 (4), 725-749.

10. Pal, M., Mather, P.M., 2005. Support vector machines for classification in remote sensing, International Journal of Remote Sensing 26 (5), 1007-1011

11. Mountrakis, G., Im, J., Ogole, C., 2011. Support vector machines in remote sensing: A review.ISPRS Journal of Photogrammetry and Remote Sensing 66 (3), 247-259 https://doi.org/10.1016/j.isprsjprs.2010.11.001

12. Camps-Valls, G., Gomez-Chova, L., Calpe-Maravilla, J., Martin-Guerrero, J.D., Soria-Olivas,E., Alonso-Chorda, L., Moreno, J., 2004. Robust support vector method for hyperspectral data classification and knowledge discovery. IEEE Transactions on Geoscience and Remote Sensing 42 (7), 1530-1542

13. Camps-Valls, G., Bruzzone, L., 2005. Kernel-based methods for hyperspectral image classification, IEEE Transactions on Geoscience and Remote Sensing 43 (6), 1351-1362.

https://doi.org/10.1109/TGRS.2005.846154

14. Guosheng Wang, A Survey on Training Algorithms for Support Vector Machine Classifiers, Department of computer science and technology, Dezhou University, Fourth International Conference on Networked
Computing and Advanced Information Management. DOI 10.1109/NCM.2008.103.

15. Ashis Pradhan, SUPPORT VECTOR MACHINE-A Survey, Computer Science and Engineering Department, Sikkim Manipal Institute of Technology, Majhitar, East-Sikkim [International Journal of Emerging Technology and Advanced Engineering, ISSN 2250-2459, Volume 2, Issue 8, August 2012].

16. Theodoros Evgenuiu and Massimilliano Pontil, Statistical Learning Theory: A Primer 1998.

17. Burges, C. J. C. (1998), A Tutorial on Support VectorMachines for Pattern Recognition. Data Mining and KnowledgeDiscovery, 2(2):121-167.

18. Guest Editorial Vapnik-Chervonenkis (VC) Learning Theory and Its Applications, IEEE TRANSACTIONS ON NEURAL NETWORKS, VOL. 10, NO. 5, SEPTEMBER 1999 985. [1045-9227(99)08541-0.] https://doi.org/10.1109/TNN.1999.788639

19. Shikha Agrawal and Jitendra Agrawal, Survey on Anomaly Detection using Data Mining Techniques, Department of Computer Science and Engineering, Rajiv Gandhi Proudyogiki Vishwavidyalaya, Bhopal, IndiaProcedia Computer Science 60 (2015) 708 - 713.

20. Andrew W. Moore and Denis Zuevy Internet Traffic Classification Using Bayesian Analysis Techniques, International conference on Measurement and modeling of computer systems, Banff, Alberta, Canada, Year of Publication: 2005, Pages: $50-60$.

21. J. Rekha, J. Bhattacharya and S. Majumder, Shape, Texture and Local Movement Hand Gesture features for Indian Sign Language Recognition, IEEE International Conference on Trendz in Information Sciences and Computing TISC 2011, 8 - 9th December,2011, Chennai, India. https://doi.org/10.1109/TISC.2011.6169079

22. N. Chandra Sekhar Reddy, Dr. Purna Chandra Rao, Dr. G Govardhan, An Intrusion Detection System for Secure Distributed Local Action Detection and Retransmission of Packets, International Journal of Soft Computing 12(1): 45-49, 2017.

23. N. Chandra Sekhar Reddy, Purna Chandra Rao Vemuri, A. Govardhan, Evaluation of PCA and K-means Algorithm for Efficient Intrusion Detection, International Journal of Applied Engineering Research ISSN 0973-4562 Volume 12, Number 12 (2017) pp. 3370-3376

24. N Chandra Sekhar Reddy, Dr. Purna Chandra Rao Vemuri, Dr. A Govardhan, Ch. Vijay, An Empirical Study On Feature Extraction Techniques For Intrusion Detection System, Journal of Advanced Research in Dynamical and Control Systems Vol. 9. Sp12, 2017. 